GEOPHYSICAL RESEARCH LETTERS, VOL. 10, NO. 5, PAGES 373-376, MAY 1983

\title{
LAMB PULSE OBSERVED IN NATURE
}

\author{
Hiroo Kanamori and Jeffrey W. Given
}

Seismological Laboratory, California Institute of Technology, Pasadena, California, 91125

Abstract. Seismograms observed at Longmire, Washington, for four eruptions of Mt. St. Helens (May 18, June 13, August 7, and August 8,1980 ), can be interpreted as Lamb pulses excited by a nearly vertical single force that represents the counter force of the eruption. These data provide reliable estimates of the impulse of the force $K$ (time integral of the force) from which the total momentum and the kinetic energy, E, of the ejecta associated with the eruption can be estimated. The values of $\mathrm{K}$ are estimated to be $1.4 \times 10^{19}, 1.4 \times 10^{16}, 3.7 \times 10^{15}$, and $2.8 \times 10^{15}$ dyne's for the four eruptions (chronological order), respectively. The corresponding values of $\mathrm{E}$ are estimated to range from 0.70 to $2.6 \times 10^{23}, 0.70$ to $2.6 \times 10^{20}, 1.9$ to $6.9 \times 10^{19}$, and 1.4 to $5.3 \times 10^{19}$ ergs using values of ejecta velocity. ranging from 100 to $375 \mathrm{~m} / \mathrm{s}$. The ratio of $\mathrm{K}$ to the amplitude of the air wave excited by the eruption is 20 to 40 times larger for the main event on May 18 than for the other events suggesting a significant difference in the eruptive mechanism. Our results demonstrate that a digital seismograph in the vicinity of volcanoes provides a simple means for quantification of the explosive power of a volcanic eruption.

Lamb (1904) computed the transient response of a homogeneous elastic half space to a single force with various geometries, and showed that a pronounced pulse propagates along the free surface with the Rayleigh-wave velocity. This pulse is preceded by minor pulses which propagate with $\mathrm{P}$ - and S-wave velocities. Here, we collectively call these pulses the Lamb pulse. Many theoretical studies on this problem have been made since then (e.g. Nakano, 1925; Lapwood, 1949; Pekeris, 1955; Chao, 1960; Burridge, 1966; Johnson, 1974; Kind, 1978; Richards, 1979).

Observationally, however, the Lamb pulse is seldom seen in its simplest form. Usually, observed seismograms are far more complex than predicted by Lamb (1904), because of the heterogeneity of the propagation medium and the complexity of the source. The source of the most naturally occurring events is represented by force couples rather than single forces. One notable exception is the observation by Benioff (1964). Benioff observed an unusual waveform from a deep earthquake in South America and argued that it was a Lamb pulse produced by a vertical single force with a step-function time history. He suggested that this single force represents a sudden collapse of a small volume of rock at the focus. Here we present examples of Lamb pulses observed in nature, and discuss its possible use for quantification of volcanic eruptions.

After the major eruption on May 18, 1980, Mt. St. Helens, Washington, erupted several more times, one of the largest being on June 13,1980. Seismic waves excited by this eruption were clearly recorded by a DWWSSN (Digital World-Wide Standardized Seismographic Network) station at Longmire (LON), a distance of about $67 \mathrm{~km}$ from the volcano. Figure 1 shows the vertical and radial component records from the long-period channel. The amplitude on the transverse component (not shown) is very small, about one-fourth of the radial component. The major pulse in the beginning of the records exhibits a phase shift of about $90^{\circ}$ between the vertical and horizontal components, and the particle motion is retrograde. These observations suggest that this pulse is a Rayleigh wave excited' by the eruption. The wavelength of this pulse is about $60 \mathrm{~km}$ which is comparable to the distance.

Copyright 1983 by the American Geophysical Union.

Paper number 3L0352.

0094-8276/83/003L-0352\$03.00
Kanamori and Given (1982) demonstrate that the massive landslide associated with the May 18, Mt. St. Helens eruption can be modeled by a nearly horizontal single force, and is responsible for the excitation of long-period $(\approx 200 \mathrm{~s})$ surface waves. On the other hand, Kanamori et al. (1983) show that the relatively shortperiod ( $5-30 \mathrm{~s}$ ) waves observed for the May 18 eruption are due to nearly vertical forces. In general, a volcanic eruption may be modeled by a combination of a single force representing the thrust of the eruption and an isotropic source corresponding to the sudden pressure release in the magma chamber. It can be shown (see Appendix to Kanamori et al., 1983) that, for a simple model of eruption, the amplitude ratio of seismic waves excited by the single force to those excited by the isotropic source is approximately equal to the ratio of the seismic-wave velocity to the particle velocity of the fluid (or gas) in the magma chamber. Since this ratio is about 10 , the source can be adequately modeled by a single force.

On these grounds, the most straightforward interpretation of the LON records is that the counter force of the eruption excited a Lamb pulse that was recorded by the seismograph after travelling the distance comparable to its wavelength. In order to investigate this problem in some detail, we compute Lamb pulses in a homogeneous half space using the expressions given by Richards (1979). A Poisson solid with a $P$ velocity of $5 \mathrm{~km} / \mathrm{s}$ is used. We take a right-handed Cartesian coordinate system with the $\mathrm{X}_{3}$ axis pointing vertically upward. The free surface is at $X_{3}=0$. We assume that the force is applied at the origin, and consider waves propagating in the positive $X_{1}$ direction. Following Richards (1979), we write the $j$-th component of displacement due to a unit step single force in the $\mathrm{i}$-th direction as $\mathrm{G}_{1 \mathrm{H}}^{\mathrm{H}}$. Then the relevant responses are: $G_{11}^{H}=I_{1} / \pi \mu r, \quad G_{1}^{H}=-G_{31}^{H}=I_{4} / \pi \mu r$, and $G_{33}^{H}-I_{3} / \pi \mu r$, where $\mu$ is the rigidity and $r$ is the distance. $I_{1}, I_{3}$, and $\mathrm{I}_{4}$ are the functions of time given by equations (10), (12), (13), (14) and (16) of Richards (1979). $\mathrm{I}_{3}$ and $\mathrm{I}_{4}$ correspond to the vertical and radial components of the displacement due to a vertical single force. Figure 2 shows $I_{3}$ and $I_{4}$ after they are convolved with the time derivative of the source time function of the form $s(t)=p /\left(p^{2}+t^{2}\right)$ that was used by Lamb (1904). Here $p=0.15 s$ is used. These functions correspond to $w_{0}$ and $q_{o}$ in Lamb's paper which are shown in the figure for comparison. Similar calculations can be made for a horizontal single force.

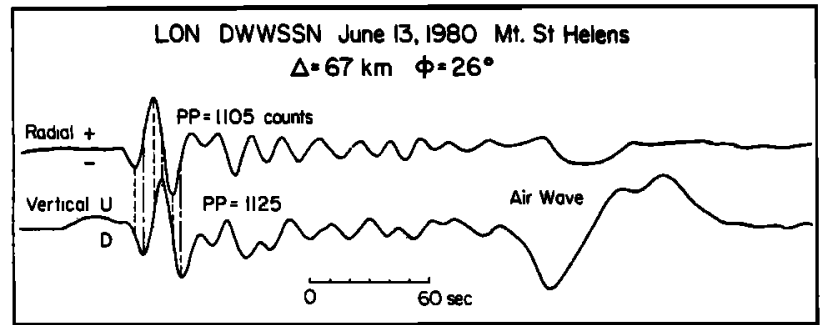

Figure 1. Seismograms of the eruption of Mt. St. Helens on June 13, 1980 recorded by a long-period digital seismograph (DWWSSN) at LON $\left(\Delta=67 \mathrm{~km}, \phi-26^{\circ}\right)$. The top trace is the radial component (upward motion indicates the ground motion away from the source) and the bottom trace is the vertical component. Note the approximately $90^{\circ}$ phase shift between the vertical and the radial component characteristic of the retrograde particle motion of Rayleigh waves. The long-period wave on the vertical component arriving about three minutes after the first pulse is the air wave excited by the eruption. At the period of $25 \mathrm{~s}$, the gain of each instrument is 500 counts/micron. 


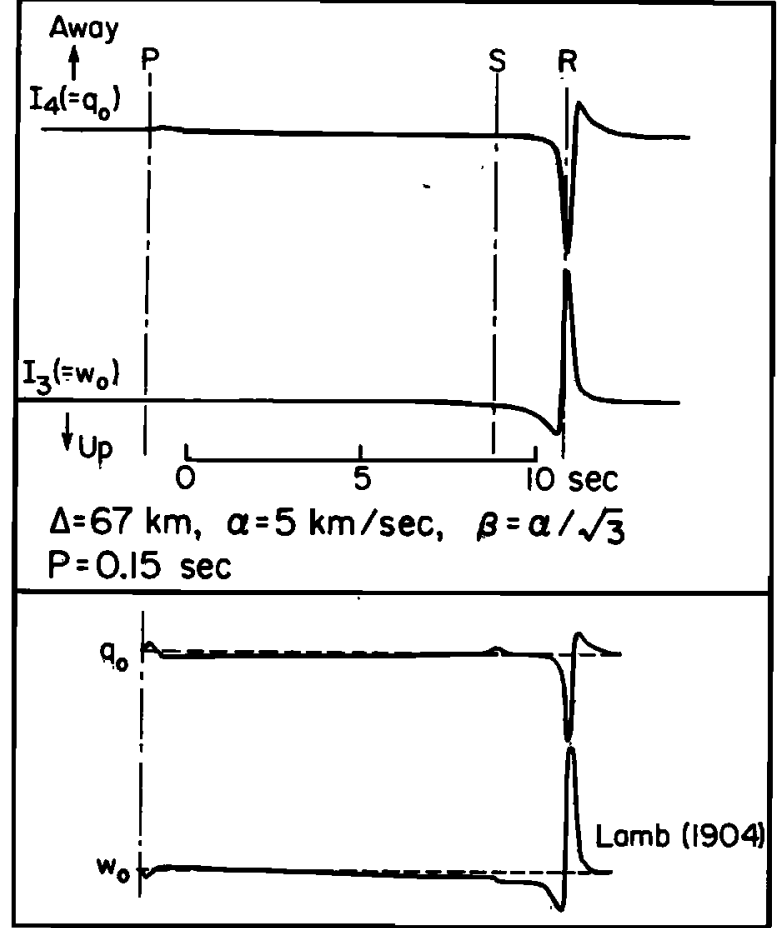

Figure 2. Lamb pulses computed for a downward vertical single force applied at the free surface of a homogeneous half-space. Richards' (1979) formulation is used. The source function is $p /\left(t^{2}+p^{2}\right)$ where $p=$ $0.15 \mathrm{~s}$. Corresponding traces given by Lamb (1904) are shown for comparison.

Next, we compute synthetic seismograms for a source time function $s(t)$ by

$$
\frac{d}{d t}\left\{I_{i}(t) * s(t) * G(t)\right\}
$$

where $G(t)$ is the seismograph response. Figure 3 shows synthetic seismograms for a symmetric triangular source time function having a total width of $5 \mathrm{~s}$. The DWWSSN long-period seismograph response is used. For a vertical single force, $\mathrm{I}_{3}$ and $\mathrm{I}_{4}$ determine the vertical and the radial.components respectively. For a horizontal single force (applied in $-X_{1}$ direction), $I_{4}$ and $I_{1}$ determine the vertical and radial components respectively. As shown by figures 2 and 3 of Richards (1979), $I_{1}$ response has a relatively large amplitude at the $\mathbf{P}$-wave arrival time. This is reflected in the large $P$ wave for the radial displacement due to a horizontal force. Comparison of Figure 3 with Figure 1 clearly suggests that the synthetics for a vertical force explain the observed waveforms and the phase lag between the vertical and horizontal components better than those for a horizontal single force, which suggests that the counter force is more vertical (downward) than horizontal. Although we cannot resolve the details of the force' geometry with the LON record alone, this result is consistent with that of Kanamori et al. (1983). Figure 4 compares the synthetics for a vertical force with the observed. The synthetics are computed using $\mathrm{r}=67 \mathrm{~km}, \mu=1.9 \times 10^{11} \mathrm{dyne} / \mathrm{cm}^{2}$, and $f_{M}$ (peak value of the force) $=5.6 \times 10^{15}$ dyne. There is some discrepancy of the waveform between the synthetics and the observed. The beginning of the synthetics represents the transient response to the $P$ wave (radial component) and the SV wave (vertical component) (see also Figure 3). The body waves are much smaller than the main Rayleigh-pulse and are comparable in amplitude to the background noise (see Figure 5). Here we are primarily concerned with the Rayleigh-wave signal, for which there is good agreement between the observed and synthetic seismograms. Also, the observed records indicate some reverberations following the main

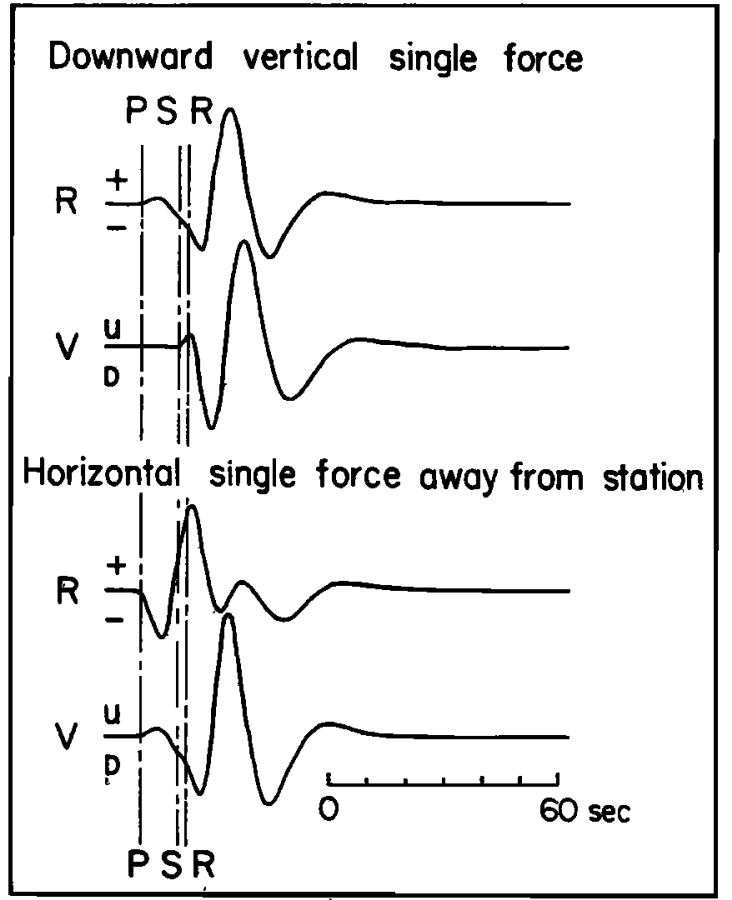

Figure 3. Synthetic seismograms computed for a vertical (downward) single force and a horizontal (away from station) single force applied at the free surface of a homogeneous half-space. A symmetric triangular source function having a total width of $5 \mathrm{~s}$ is used. The response of the DWWSSN long-period seismograph is convolved with the ground displacement. The distance is $67 \mathrm{~km}, P$ velocity is $5 \mathrm{~km} / \mathrm{s}$ and the Poisson's ratio is 0.25 . Note the relatively large $P$ wave on the radial component for a honzontal force.

pulse. This discrepancy is probably due to the dispersion during propagation and the complexity of the source time history, but we do not pursue this point in view of the limitation of the single station data.

In the present calculation, a total width of $5 \mathrm{~s}$ is used for the symmetric source time function. Because of the relatively narrow

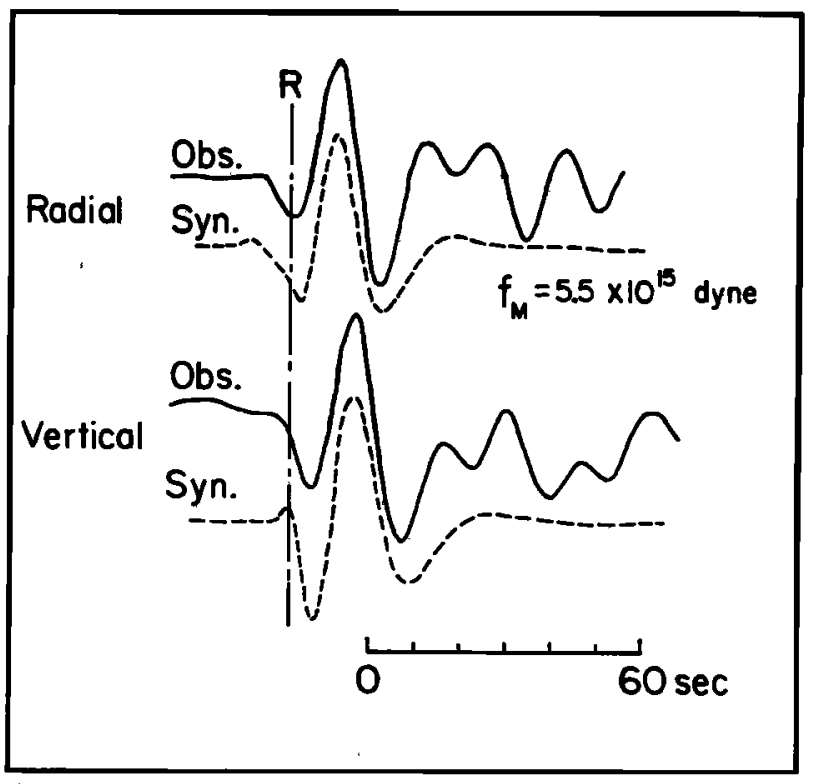

Figure 4. Comparison of the observed and synthetic seismograms. The dashed line indicates the arrival time of the Rayleigh pulse. 
bandwidth of the instrument response, we cannot determine this value very well. A width of $10 \mathrm{~s}$ makes the synthetic too broad, but any value less than $5 \mathrm{~s}$ yields an adequate fit. Comparing the amplitude of the synthetic with the observed, we estimate the strength of the single force. If the duration of the source time function is shorter than the characteristic period of the instrument, here about $25 \mathrm{~s}$, the amplitude of the synthetic is approximately proportional to the impulse of the force $K=\int_{0}^{f} f(t) d t$. Our numerical experiment shows that the amplitude variation is \pm 16 $\%$ around the average for $1 \leqslant \tau \leqslant 15 \mathrm{~s}$. In our case $\mathrm{K}=\mathrm{f}_{\mathrm{M}} \tau / 2$ where $f_{M}$ is the peak value of the force and $\tau$ is the total width of the triangular function. Comparing the amplitude, we estimate $\mathrm{f}_{\mathrm{M}} \tau / 2=1.4 \times 10^{16}$ dyne.s. If $\tau$ is $5 \mathrm{~s}, \mathrm{f}_{\mathrm{M}}=5.5 \times 10^{15}$ dyne.

These estimates depend on the geometry of the source and its time history. Since our model is a very simple one, and details cannot be resolved, these values should be considered only approximate; they are probably uncertain by a factor of two or so.

Similar Lamb pulses were observed for the main eruption on May 18, 1980, and the smaller eruptions on August 7 and 8 , 1980. For a relatively large eruption on July 22,1980 , no record is available. Figure 5 compares these records. The waveform of the main eruption is very complex compared with the others. This complexity is due to the complex eruptive sequence associated with the May 18 event. Kanamori et al. (1983) analyzed farfield $P$ waves excited by this event and conclude that the source is represented by a series of vertical single forces with a peak value of about $2.6 \times 10^{17}$ dyne. The characteristic period of the seismogram for the main event is about $25 \mathrm{~s}$ and is significantly longer than that of the other events (Figure 5). In order to explain this period, the width of the source time function, expressed by a symmetric triangular function, must be about $25 \mathrm{~s}$. The characteristic periods of the August 7 and 8 events are about the same as that of the June 13 event. From the amplitude, we estimate $\mathrm{K}-\mathrm{f}_{\mathrm{M}} \tau / 2=3.7 \times 10^{15}$ dyne's and $2.8 \times 10^{15}$ dyne's for the August 7 and August 8 events respectively. For the main event, the waveform is very complex and long. If we use the time history given by Kanamori et al. (1983), the impulse $\mathrm{K}$ is approximately $1.4 \times 10^{19}$ dyne-s, about 3 orders of magnitude larger than that for the June 13 event. The results are summarized in Table 1.

Figure 5 also shows air waves excited by the eruptions. Since the DWWSSN seismograph system is not air-tight, it responds to

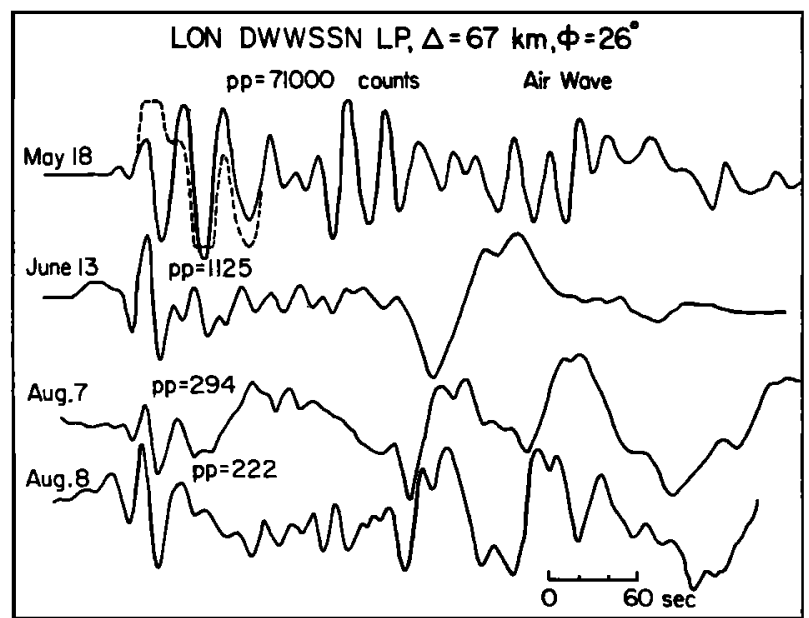

Figure 5. Comparison of the DWWSSN long-period seismograms (vertical component) for the four major eruptions. A portion of the longperiod record for the May 18 event went off scale and is indicated by a dotted curve. The displacement record for this part is obtained from the intermediate-period channel after the difference in the response is corrected. Note the complexity of the waveform of the main eruption. The gain of the instrument is 500 counts/micron at $T=25 \mathrm{~s}$.
Table 1.

The impulse of the force, $K$, determined from the Lamb pulse.

\begin{tabular}{lrlccc}
\hline \multicolumn{2}{c}{ Event } & $\begin{array}{c}\mathbf{K} \\
(\text { dyne-s) }\end{array}$ & $\begin{array}{c}\boldsymbol{\tau} \\
(\mathrm{s})\end{array}$ & $\begin{array}{c}\mathbf{f}_{\mathrm{M}}^{*} \\
(\text { dyne) }\end{array}$ \\
\hline May & 18 & $15: 32$ & $1.4 \times 10^{19}$ & 25 & $2.6 \times 10^{17}$ \\
June & 13 & $04: 10$ & $1.4 \times 10^{16}$ & $>5$ & $>5.6 \times 10^{15}$ \\
Aug. & 7 & $23: 26$ & $3.7 \times 10^{15}$ & $<5$ & $>1.5 \times 10^{15}$ \\
Aug. & 8 & $05: 32$ & $2.8 \times 10^{15}$ & $<5$ & $>1.1 \times 10^{15}$ \\
\hline
\end{tabular}

${ }^{*} f_{M}$ is calculated by $f_{M}=2 K / \tau$, for all events except the May 18 eruption. For the May 18 event, $f_{M}$ and $\tau$ taken from Kanamori et al. (1983) are used to calculate $\mathrm{K}$.

the change in the air density associated with the pressure change. It can be easily shown that the pressure change $\Delta P$ produces the same response as the ground displacement

$$
\mathrm{y}=\frac{\mathrm{T}^{2} \mathrm{~g}}{4 \pi^{2}} \cdot \frac{\rho}{\rho_{\mathrm{M}}} \cdot \frac{l_{\mathrm{v}}}{l_{\mathrm{g}}} \cdot \frac{\Delta \mathrm{P}}{\mathrm{P}}
$$

where, $\mathrm{T}$ is the period, $\mathrm{g}$ is the acceleration of gravity, $\rho$ is the density of the air, $P$ is the atmospheric pressure and $\rho_{M}$ is the average density of the effective mass of the seismograph. $l_{\mathrm{v}}$ is the distance between the pivot of the pendulum and the center of volume of the effective mass, and $l_{\mathrm{g}}$ is the distance between the pivot and the center of gravity of the effective mass. Using the physical parameters appropriate for the DWWSSN instrument, we have approximately

$$
\Delta \mathrm{P} / \mathrm{P}=806 \mathrm{y} / \mathrm{T}^{2}
$$

where $\mathrm{y}$ is in $\mathrm{cm}$ and $\mathrm{T}$ is in $\mathrm{s}$. We estimate the amplitude of the air waves using this relation. For the main event, the high frequency component is filtered out. For the August 7 and 8 events, only the first cycle is used. The results are summarized in Table 2.

We note that the ratio $\eta=K / \Delta P$ is about 20 to 40 times larger for the main eruption than for the later eruptions. This difference may be due to the difference in the eruption mechanism. The main eruption was associated with the massive landslide which uncapped the vent, while other eruptions took place without it. However, the details of the mechanism of air wave excitation need to be investigated before we can fully understand the reason for this difference.

We have shown that the Lamb pulse can be used to determine the impulse of the force, $\mathrm{K}$, associated with the eruption. The impulse $\mathbf{K}$ is a very useful parameter for quantification of volcanic eruptions, because it can be related to various physical parameters such as the kinetic energy $E$ and the pressure $P$. We let the total mass of the ejecta, the initial velocity, and the cross sectional area of the vent be $M, v_{0}$ and $S$ respectively. Then $\mathrm{E}=(1 / 2) \mathrm{Mv}_{0}^{2}$ and $\mathrm{K}=\mathrm{Mv}_{0}$, from which we have $\mathrm{E}=(1 / 2) \mathrm{v}_{0} \mathrm{~K}$. Since the estimate of the initial velocity of ejecta ranges from 100 to $375 \mathrm{~m} / \mathrm{s}$ (Kieffer, 1981; Friedman et al., 1981; Eichelberger and Hayes, 1982), the kinetic energy is estimated to range from 0.70 to $2.6 \times 10^{23}, 0.70$ to $2.6 \times 10^{20}, 1.9$ to $6.9 \times 10^{19}$, and 1.4 to $5.3 \times 10^{19}$ ergs for the May 18, June 13, August 7 and August 8 eruptions respectively. The estimate of the pressure $P$ can be made from $f_{M}$ if $S$ is known. For the main event, if $S$ is $2.5 \times 10^{9} \mathrm{~cm}^{2}$ (the value used by Kieffer, 1981), then $P$ is estimated to be 104 bar which is approximately equal to the overburden pressure due to the landslide mass.

Since the partition of energy between elastic-wave energy, airwave energy and other types of energy (e.g. potential energy, thermal energy etc.) depends upon the mechanism of the eruption and the type of the volcano (e.g. andesitic versus basaltic), the measurement of Rayleigh waves alone provides only partial quantification of the eruption. Nevertheless, the method presented here provides a very simple means for quantification of the explosive power of volcanoes. 
Table 2. Air-wave data.

\begin{tabular}{lrcccc}
\hline \multicolumn{1}{c}{ Event } & $\begin{array}{c}\mathrm{T} \\
(\mathrm{s})\end{array}$ & $\begin{array}{c}\text { Amp. (p-p) } \\
(\text { counts) }\end{array}$ & $\begin{array}{c}\text { Equivalent } \\
\text { Ground Motion } \\
(\mu)\end{array}$ & $\begin{array}{c}\Delta \mathrm{P} \\
(\mathrm{mbar})\end{array}$ & $\begin{array}{c}\mathrm{K} / \Delta \mathrm{P} \\
10^{13} \\
\mathrm{~s} \cdot \mathrm{cm}^{2}\end{array}$ \\
\hline May 18, 1980 & 144 & 20,000 & 2,000 & 7.6 & 180. \\
June 13, 1980 & 108 & 1,278 & 32 & 0.22 & 4.5 \\
Aug. 7, 1980 & 60 & 470 & 2 & 0.045 & 8.2 \\
Aug. 8, 1980 & 52 & 240 & 1 & 0.030 & 9.3 \\
\hline
\end{tabular}

Acknowledgment. We thank Robert Engdahl and John Hoffman for providing us with the LON digital seismogram tapes used in this study. Tom McEvilly gave us useful data necessary for computing the response of the DWWSSN instrument to air-waves. This research was partially supported by National Aeronautics and Space Administration grant number NSG-7610, and National Science Foundation grant EAR811-6023. Contribution No. 3843, Division of Geological and Planetary Sciences, California Institute of Technology, Pasadena, California 91125.

\section{References}

Benioff, H., 1964, Earthquake source mechanisms, Science, 143, 1399-1406.

Burridge, R., 1966, A new look at Lamb's problem, J. Phys. Earth, I6, Special Issue, 169-171.

Chao, C.-C., 1960, Dynamical response of an elastic half-space to tangential surface loadings, $J$ Appl Mech., 27, 559-567.

Eichelberger, J. C., and D. B. Hayes, 1982, Magmatic model for the Mount St. Helens blast of May 18, 1980, J. Geophys. Res., 87, 7727-7738.

Freidman, J. D, G. R. Olhoeft, G. R. Johnson, and D. Frank, 1981, Heat content and thermal energy of the June dacite dome in relation to total energy yield, May - October 1980, US Geol Surv Prof Pap. 1250, 557-567.
Johnson, L. R., 1974, Green's function for Lamb's problem, Geophys. J. R. astr. Soc., 37, 99-131.

Kanamori, H., J. W. Given, and T. Lay, 1983, Analysis of seismic body waves excited by the Mt. St. Helens eruption of May 18, 1980, in preparation.

Kieffer, S. W., 1981, Blast dynamics at Mt. St. Helens on 18 May 1980, Nature, $291,568-570$.

Kind, R., 1978, The reflectivity method for a buried source, Geophys $J$. R. astr. Soc., 44, 603-612.

Lamb, H., 1904, On the propagation of tremors over the surface of an elastic solid, Phil. Trans. $R$. Soc. (London) A, 203, 1-42.

Lapwood, E. R., 1949, The disturbance due to a line source in a semiinfinite elastic medium, Phil. Trans. Roy. Soc. (London) $A, 242$, 841, 63-100.

Nakano, H., 1925, On Rayleigh waves, Japan J. Astron. Geophys., 2, 233-326.

Pekeris, C. L., 1955, The seismic surface pulse, Proc. Natl. Acad Sci., U.S., $41,469-480$

Richards, P. G., 1979, Elementary solutions to Lamb's problem for a point source and their relevance to three-dimensional studies of spontaneous crack propagation, Bull. Setsmol. Soc. Am., 69, 947-956.

(Received November 16, 1982;

revised February 16, 1983; accepted February 22, 1983.) 